\title{
Aalto-1 nanosatellite - technical description and mission objectives
}

\author{
A. Kestiläa ${ }^{1}$, T. Tikka ${ }^{1}$, P. Peitso ${ }^{1}$, J. Rantanen ${ }^{1}$, A. Näsilä ${ }^{1}$, K. Nordling ${ }^{1}$, H. Saari ${ }^{2}$, R. Vainio ${ }^{3}$, P. Janhunen ${ }^{4}$, \\ J. Praks ${ }^{1}$, and M. Hallikainen ${ }^{1}$ \\ ${ }^{1}$ Aalto University, Department of Radio Science and Engineering, P.O. Box PL13000 Aalto, 00076 Espoo, Finland \\ ${ }^{2}$ VTT Optical Instruments, P.O. Box 1000, 02044 VTT Espoo, Finland \\ ${ }^{3}$ Helsinki University, Department of Physics, P.O. Box 64, 00014 Helsinki, Finland \\ ${ }^{4}$ Finnish Meteorological Institute, Space Research, P.O. Box 503, 00101 Helsinki, Finland
}

Correspondence to: A. Kestilä (antti.kestila@aalto.fi)

Received: 12 September 2012 - Published in Geosci. Instrum. Method. Data Syst. Discuss.: 26 November 2012

Revised: 29 January 2013 - Accepted: 30 January 2013 - Published: 21 February 2013

\begin{abstract}
This work presents the outline and so far completed design of the Aalto-1 science mission. Aalto-1 is a multi-payload remote-sensing nanosatellite, built almost entirely by students. The satellite aims for a $500-900 \mathrm{~km}$ sunsynchronous orbit and includes an accurate attitude dynamics and control unit, a UHF/VHF housekeeping and S-band data links, and a GPS unit for positioning (radio positioning and NORAD TLE's are planned to be used as backup). It has three specific payloads: a spectral imager based on piezoactuated Fabry-Perot interferometry, designed and built by The Technical Research Centre of Finland (VTT); a miniaturised radiation monitor (RADMON) jointly designed and built by Universities of Helsinki and Turku; and an electrostatic plasma brake designed and built by the Finnish Meteorological Institute (FMI), derived from the concept of the e-sail, also originating from FMI. Two phases are important for the payloads, the technology demonstration and the science phase. The emphasis is placed on technological demonstration of the spectral imager and RADMON, and suitable targets have already been chosen to be completed during that phase, while the plasma brake will start operation in the latter part of the science phase. The technology demonstration will be over in a relatively short time, while the science phase is planned to last two years. The science phase is divided into two smaller phases: the science observations phase, during which only the spectral imager and RADMON will be operated for 6-12 months and the plasma brake demonstration phase, which is dedicated to the plasma brake experiment for at least a year. These smaller phases are necessary due to the drastically different power, communication and attitude requirements of the payloads. The spectral imager will be by
\end{abstract}

far the most demanding instrument on board, as it requires most of the downlink bandwidth, has a high peak power and attitude performance. It will acquire images in a series up to at least 20 spectral bands within the $500-900 \mathrm{~nm}$ spectral range, forming the desired spectral data cube product. Shortly before an image is acquired, the parallel visual spectrum camera will take a broader picture for comparison. Also stereoscopic imaging is planned. The amount of data collected by the spectral imager is adjustable, and ranges anywhere from 10 to $500 \mathrm{MB}$. The RADMON will be on $80 \%$ of an orbit period on average and together with housekeeping data will gather around $2 \mathrm{MB}$ of data in $24 \mathrm{~h}$. An operational limitation is formed due to the S-band downlink capability of $29-49 \mathrm{MB}$ per $24 \mathrm{~h}$ for a $500900 \mathrm{~km}$ orbit altitude, as only one ground station is planned to be available for the satellite. This will limit both type and quantity of spectral imager images taken during the science phase. The plasma brake will in turn be within an angle of $20^{\circ}$ over the poles for efficient use of the Earth's magnetic field and ionosphere during its spin-up and operation.

\section{Introduction}

Aalto-1 is a student nanosatellite with three specific payloads that aim for tests proving their capabilities in an actual space application. The satellite itself and its mission have been designed particularly keeping in mind the aims and needs of the payloads, nevertheless conforming as much as possible to the CubeSat-specifications and the learned practices of the CubeSat community. The CubeSat-specifications (CDS, 


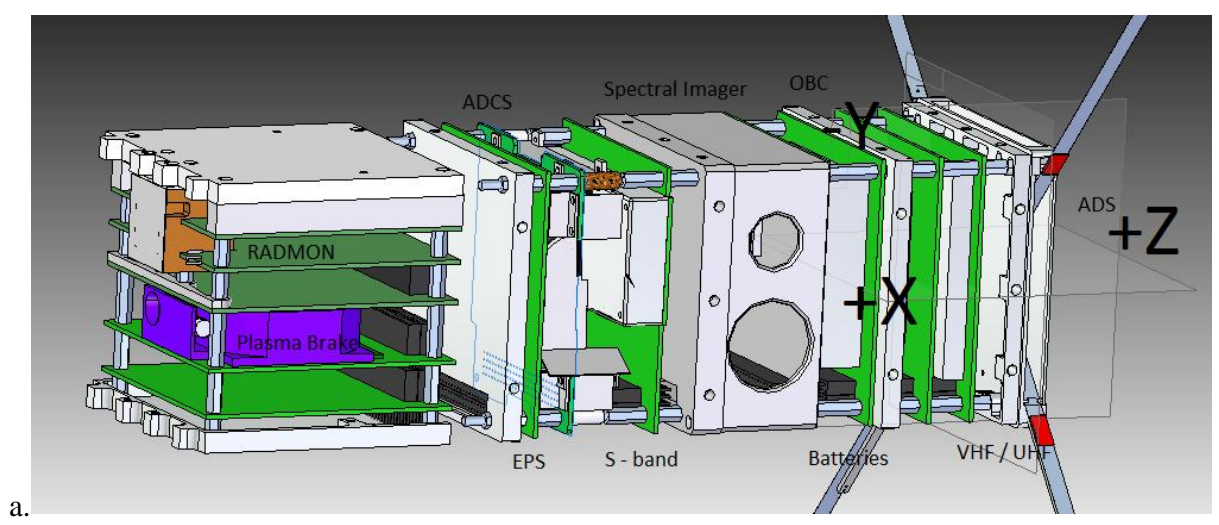

a.

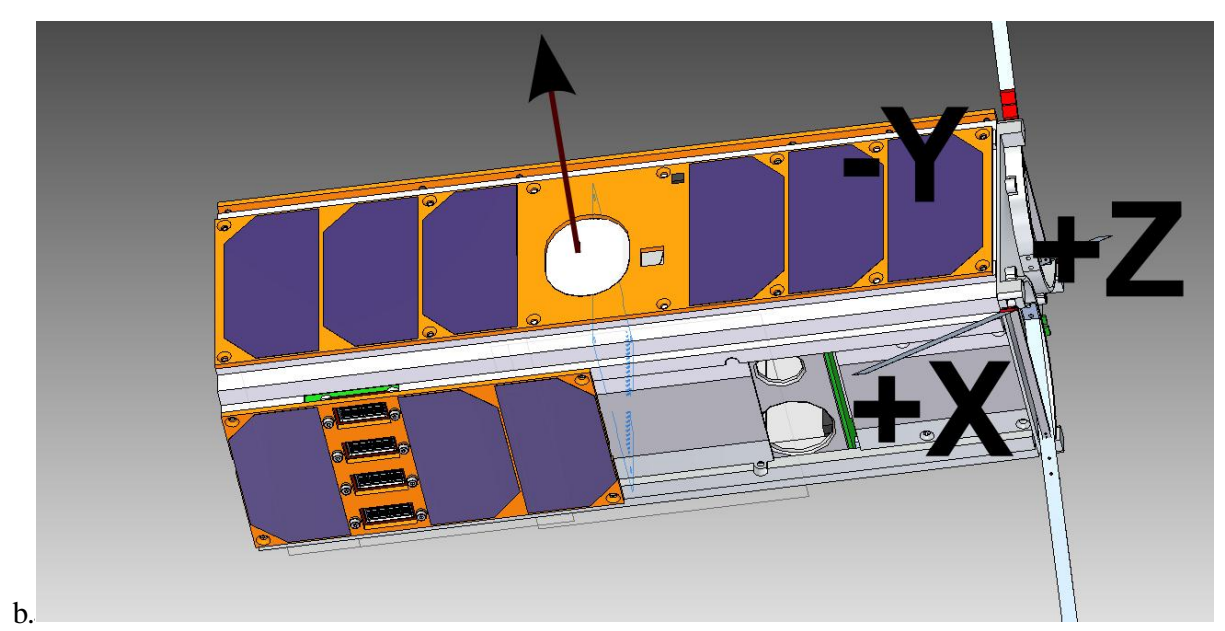

Fig. 1. (a) A exposure of the Aalto-1 subsystems, in which EPS is the electrical power system, OBC the onboard computer and ADS the antenna deployment system (GPS receiver will be integrated with the S-band board). (b) The satellite's external dimensions are $10 \times 10 \times 34 \mathrm{~cm}$. (The position of ADCS star tracker is at the centre of the - Y-side alongside the smaller GPS antenna, represented here by the bigger and smaller holes, while the electron guns are at the left side of the $+X$-side.) The satellite's velocity vector will be parallel to its $-\mathrm{Y}$ direction.

2009 themselves are a set of guidelines jointly developed by California Polytechnic State University and Stanford University, which enable the standardisation of the satellite bus, mechanical and launch properties. The first part of the actual mission of the satellite consists of remote sensing using a spectral imager as well as orbital science observations with a radiation monitor, while during the latter part an experiment on determining the drag force created by an electrostatic plasma brake will be performed.

While the goal of this student project is gathering experience and so most of the satellite's subsystems will be built in-house by students, nevertheless thanks to conformity with specifications, many of the needed subsystem can and will be bought as COTS from external manufacturers. This work presents the mission in its entirety, as well as in more detail the technology demonstration and scientific phases of the mission. The Aalto-1 nanosatellite will perform a complicated mission both in terms its technological and scientific goals, and the mission technical requirements derived from them. As such it demands well-performing subsystems to track, handle attitude demands, process and downlink in one seamless whole to succeed in attaining these goals. A dynamic attitude in handling will especially be required, as the mission is divided into two clear cut phases, and needs to be accurate as well as flexible.

\section{Technical overview of Aalto-1}

Aalto-1 is a $3 \mathrm{U}$ nanosatellite, and conforms to the CubeSatspecifications (see Fig. 1). The satellite has a mass of $4 \mathrm{~kg}$ and external dimensions of $10 \times 10 \times 34 \mathrm{~cm}$, designed to fit into a Poly-PicoSatellite Orbital Deployer (P-POD).

The mechanical structure of the satellite consists of two subsystem PCB fastener stacks, conjoined specially designed stack plates to both each other and the main outer frame. The outer frame itself consists of two separate aluminium parts, both $1.5 \mathrm{~mm}$ thick, fastened together into one tubelike structure. 

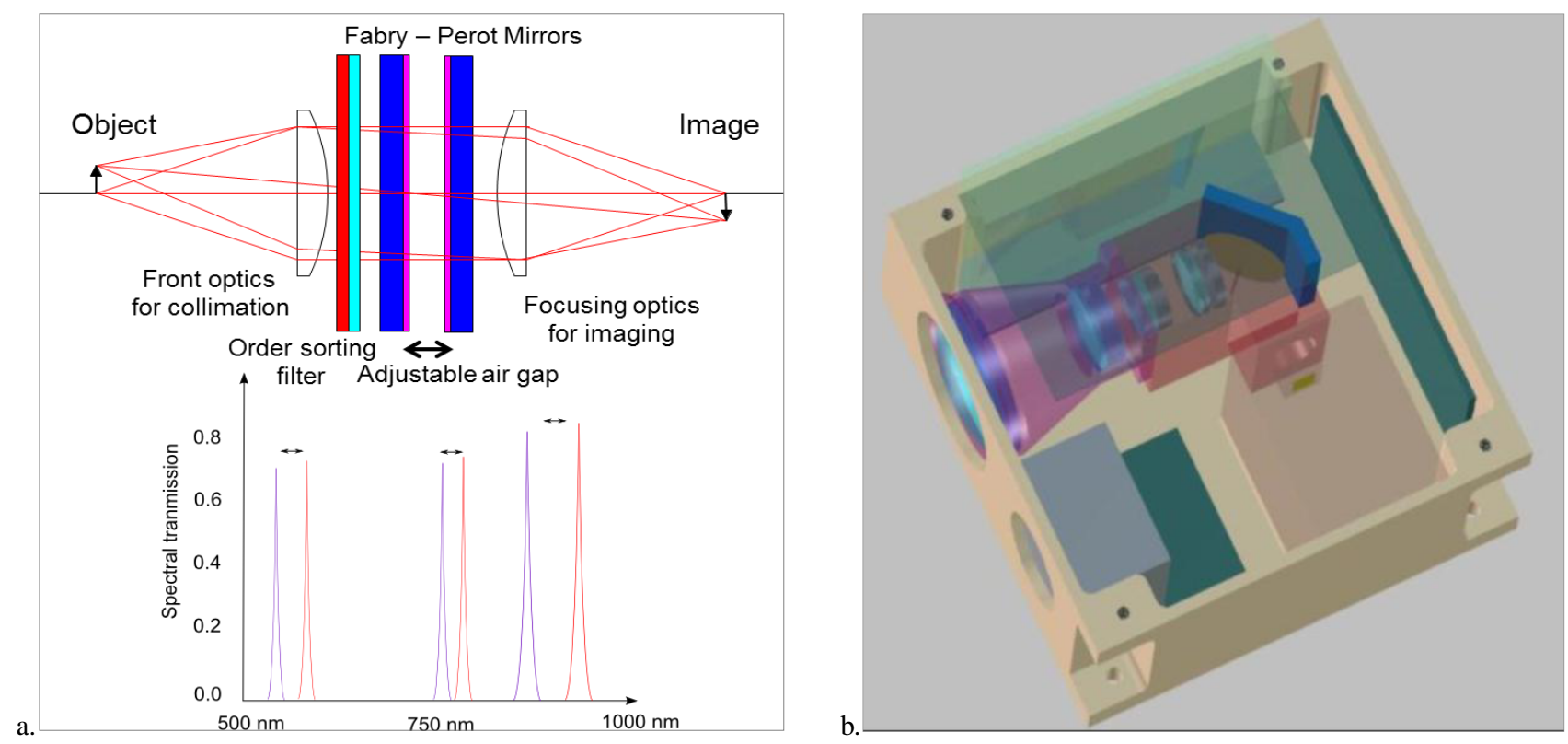

Fig. 2. (a) The Fabry-Perot interferometer concept at work; the change in the Fabry-Perot mirror interdistance causes a change in the spectral bandwidth to the imaging CMOS camera. (b) Structural view of the spectral imager instrument, the larger instrument representing the spectral imager, while the smaller (beneath) instrument is the VIS-camera (Näsilä et al., 2012).

The satellite orbit is not yet finalised, as a contract with the launcher has not been signed. During launch the satellite will be sealed within the special, CubeSat-specification conforming deployment pod. This pod is designed to protect (the satellite within as well as the launcher and surrounding satellites) and when the time is correct, deploy the satellite to its correct orbit. The ideal orbit is a sun-synchronous "middaymidnight" orbit, with an orbit altitude of $500-900 \mathrm{~km}$. The orbit can have a 10:30-13:30 MLT (mean local time) variation, with an otherwise as standard as possible configuration in order to find a suitable launch easier. Once the satellite is deployed out of its CubeSat-deployer, it will remain in the same orbit throughout the first part of its science mission. During the latter part, however, as the plasma brake experiment is active, its orbit altitude and so other parameters as well will change thanks to the drag force caused by the tether. Due to the uncertainty in the final orbit, the various effects of the orbit parameters, such as power production and thermal environment, have been designed with a $20 \%$ margin, and so the mission can continue in a sun-synchronous orbit with a significantly differing beta angle. The satellite will be covered with solar cells on almost all available surfaces, forming three long-side solar panels, and one smaller on the nadir side (during the remote-sensing phase of the mission). These panels produce roughly an average power of $4.5 \mathrm{~W}$ for an ideal $500 \mathrm{~km}$ orbit in the entirety of the mission, with a battery as part of the electrical power system to store the collected energy for the eclipse period in the orbit.

The satellite will have three radio channels in use, of which VHF/UHF will be used for up/downlinking telemetry, tracking and commands, and S-band will be only transmitting data down to the ground segment. Depending on the orbit altitude, the S-band will be able to downlink 29 to $49 \mathrm{MB}$ per $24 \mathrm{~h}$, while VHF/UHF can uplink 0.07 to $0.12 \mathrm{MB}$ per $24 \mathrm{~h}$ (with a $1200 \mathrm{bps}$ uplink) and downlink 0.57 to $0.96 \mathrm{MB}$ per $24 \mathrm{~h}$ (downlink $9600 \mathrm{bps}$ ), and can work as a limited backup for the S-band.

The onboard computer (OBC) has been designed in-house, and will employ a Linux real-time operating system with a double-redundant design (two computers on one PCB-board) and necessary flash memory needed for storing extensive spectrometer payload imagery.

The satellite will have attitude dynamics and a control system (ADCS), designed and built by an external manufacturer Berlin Space Technologies. The iADCS-100 is a semiautonomous ADCS and will have attitude knowledge and pointing accuracy of less than a degree during the science observation phase and less than $10^{\circ}$ during plasma brake operation, with several operation modes for the entire mission, with only rough positional information required. The system will consist of magnetorquers, MEMS gyros, miniature reaction wheels, sun sensors and a star tracker for accurate attitude knowledge. It will fit into a less than half a CubeSatcube space and have several operational modes, with the only external requirement being positional knowledge every few days.

During the mode requiring high accuracy attitude knowledge, the star tracker will be the primary source of knowledge, with the reaction wheels enabling precise pointing, while the magnetorquers providing the "raw" and less precise 
pointing. The latter phase of the mission, during which only the plasma brake will be operated, will use only the magnetorquers, MEMS gyros and the sun sensors for attitude purposes.

For gathering positional knowledge, an IT03 GPS receiver by Fastrax will be employed primarily, with a backup of radio positioning using ranging and Doppler distortion, as well as NORAD supplied TLE's. The GPS will be operated relatively rarely and will be operational either just before the spectral imager takes an image, and later during the plasma brake experiment, once per orbit. This is mainly to conserve the satellite's power budget, as the precise orbit position is not necessary except when determining the satellite's position during imaging by post-processing the GPS data. Later, the altitude change due, to the use of the plasma brake, is expected to be low enough for a once per orbit determination to be enough, thus, saving power and downlink budget. By default in all cases, the GPS will be cold-started each time, taking a few minutes for total time of the first fix (based on tests performed on it), after which 60 fixes will be acquired (one fix per second).

The satellite will have to operate in a heavy radiation and thermal environment for a long time period, necessitating stringent test campaigns on all of its onboard subsystems, performed according to ECSS standards.

The main planned test campaigns planned are:

- Thermal cycling on all subsystems indicated to experience critical temperature variations by thermal analysis.

- As the satellite will have lenses, vacuum degassing and testing on all materials with a risk of outgassing will be performed.

- Radiation testing on radiation sensitive subsystems, such as the OBC, will be performed to make sure the system will have the intended lifetime.

- Extensive qualification thermal vacuum tests on the whole satellite system during verification.

Testing of some subsystems has already begun, with the first version of the onboard computer as well as the COTS GPS receiver successfully cycled through a -65 to $+90^{\circ}$ extreme range thermal cycle. The first versions of the solar cell panels have also gone through a space-grade vacuum, with the purpose of determining the outgassing level of the silicone used in attaching the cells on the panels.

In addition, functional testing has already been started on all first draft electrical models available of the satellite payloads.

\section{Overview of Aalto-1 payloads}

Three specific payloads are carried by the satellite, each of them having a very different mission profile and scientific target. Here the technical details and scientific motivation for these payloads are described in more detail.

\subsection{Aalto-1 spectral imager}

The Aalto-1 spectral imager is intended primarily as a technology demonstration and, if operating successfully, will be used for actual scientific remote-sensing purposes. The imager is developed by the Technical Research Center of Finland (VTT), uses miniaturised interferometry technology in the form of adjustable Fabry-Perot mirrors, supporting optics and an RGB CMOS sensor, with an auxiliary VIScamera confirming the location imaged and whether the image is worth downlinking (there might be cloud cover, etc.). The sensor takes a spatial image in 1 to 3 wavelength channels simultaneously within the 500-900 nm bandwidth, and using an exposure time of $10-20 \mathrm{~ms}$ per individual image, a hyperspectral datacube consisting of 20-60 channels can be recorded in less than $1.5 \mathrm{~s}$ (Saari et al., 2009; US Patent US 8, 130, 308 B2, 2012; Mannila et al., 2011) (see Fig. 2). The gap between the Fabry-Perot mirrors is controlled by three piezo-actuators in a closed capacitive feedback loop (Antila et al., 2010). The sensor will have a field-of-view of $10 \times 10^{\circ}$, while the additional VIS-camera will have a $30 \times 19^{\circ}$. At an orbit altitude of $700 \mathrm{~km}$, a swath width of $120 \mathrm{~km}$ (along and cross), a target signal-to-noiseratio (SNR) of 50 and a spatial resolution of $240 \mathrm{~m}$ can be achieved, while the spectral resolution will be in the range of 10 to $30 \mathrm{~nm}$. The SNR can be modified using pixel binning, with a nominal and maximum image size of $512 \times 512$ and $2048 \times 2048$ pixels, respectively. As with the amount of wavelength channels can be chosen, the data size can also vary from $2 \mathrm{MB}$ to an absolute maximum of $500 \mathrm{MB}$. The command of the spectrometer will be performed by the onboard computer through I2C, while the image data will be sent through 3-wire SPI to the onboard computer's dedicated buffer memory, and onwards eventually to the downlinking S-band radio.

Initially, the images taken by the spectrometer will be evaluated to determine the instrument's performance in space after an actual launch and the subsequent space conditions. After successfully determining the imager's operation and performance, the instrument will be used in a remote-sensing campaign, currently in planning, as well as for stereoscopic imaging of selected targets in order to prove the successful use of such an imaging method with a nanosatellite. The instrument itself is not intended as a competition to existing spectral imagers in terms of image quality, especially in terms of ground pixel resolution, but rather to prove that the central technology, the Fabry-Perot mirrors within it, work properly in space. 


\subsection{Miniaturized particle telescope RADMON}

Aalto-1 will chart Earth's LEO proton (10-200 MeV) and electron $(0.7-10 \mathrm{MeV})$ radiation environment with the use of its second payload, the miniature particle telescope RADMON, developed by University of Helsinki and Turku. The telescope is made out of a thin silicon detector measuring incoming proton or electron particle energy loss, and a thicker caesium scintillator combined with a photodiode for measuring the leftover energy in the particles caught in the telescope (see Fig. 3). With the help of these two signals, the particle species can be determined as well as their respective incoming (before hitting the telescope) energies. The measurements themselves consist of particle counting rates (i.e., fluxes) in several energy pass bands inside the available energy range. The differential energy from the detector and scintillator channels with $\Delta E / E \sim 50 \%(\sim 5$ channels per decade), mainly to allow the comparison with other instruments on selected energy bands. With a nominal $15 \mathrm{~s}$ time resolution (corresponding to $1^{\circ}$ in latitude on a 90min orbit period), integral fluxes down to $1 \mathrm{~cm}^{2} \mathrm{sr}^{-1} \mathrm{~s}^{-1}$ should be detectable, while its dynamic range goes up to $10^{5} \mathrm{~cm}^{2} \mathrm{sr}^{-1} \mathrm{~s}^{-1}$. As seen in Fig. 3b, the telescope consists of a detector unit (the brown box in front), the analogue board responsible for amplifying the detector unit signals and digitising them, digital board with an FPGA for signal processing and conversion in final format, and finally the power supply unit.

RADMON will have UART as its primary data and command channel to the OBC, with I2C working as backup, which is sufficient for the estimated total data of $1 \mathrm{MB}$ per $24 \mathrm{~h}$ of operation.

As with in the case of the imager, the primary goal of RADMON is demonstrating that the instrument technology will survive the launch and subsequent space conditions. Once that is achieved, RADMON will proceed with its aimed scientific goals (see Sect. 4.1).

\subsection{Electrostatic plasma brake}

The third satellite payload, the electrostatic plasma brake, designed and built by the Finnish Meteorological Institute, is based on the so-called e-sail concept, developed to enable fast and cheap interplanetary travel (Janhunen et al., 2010). The main purpose of the plasma brake is to demonstrate the physical concept behind this e-sail concept, as it remains unproven so far. The tether intends to create a Coulomb drag force and so eventually, during its phase of the mission, change the satellite's orbital velocity and, thus, the altitude. As the aim is to prove that the concept works, the orbit parameters need to be only roughly determined, with the planned use of GPS once per orbit during the plasma brake experiment.

The plasma brake consists of a long, positively or negatively charged tether and support subsystems (Janhunen et
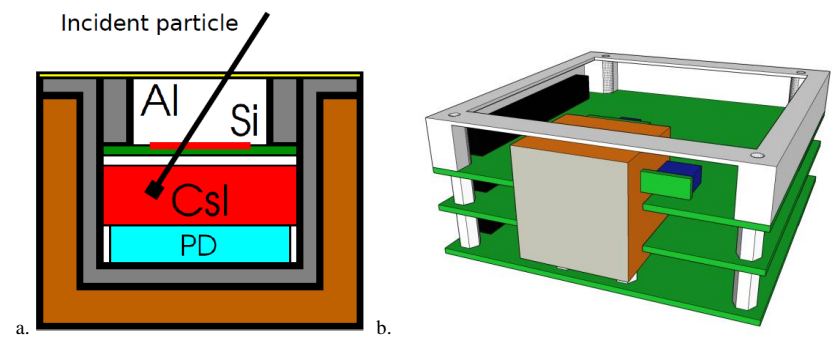

Fig. 3. (a) A cut of the RADMON detector unit showing the $\mathrm{Si}$ detector (blue chip) and the $\mathrm{CsI}(\mathrm{Tl})$ scintillator (red cube). Particles enter the system through the 300-mum thick Al window (grey) and a signal from both detectors is required for counting the particle. The housing (brown) is made of brass. (b) Sketch of the RADMON unit including the stack of three PCBs (analogue, digital and power supply, from top to down) and the detector unit in the front (grey, brown box).

al., 2010), and the tether reacts with the incoming ionosphere in orbit, creating a Coulomb drag force as charged particles interacting with the charged tether (see Fig. 4a), as the spacecraft is moving with respect to the ionosphere.

Once the plasma brake mission phase begins, the tether will be reeled out with a speed of $1-3 \mathrm{~mm} \mathrm{~s}^{-1}$, and will have a weight of 0.5 to $1 \mathrm{~g}$ at its tip to facilitate a smoother reelout. The satellite at this point will be spun up to assist during the deployment process.

The tether itself will be $100 \mathrm{~m}$ long and $25-50 \mu \mathrm{m}$ wide, consisting of several smaller aluminium strands interwoven such that the risk of the tether snapping is minimised (see Fig. 4b). Two operating modes are possible, depending on whether the tether is positively or negatively charged, with the former mode requiring a special electron gun, currently being developed in the University of Jyväskylä. The electron gun is intended to neutralise the positively charged plasma brake and, thus, close the circuit with respect to the surrounding plasma of the ionosphere.

The plasma brake consists of three specific PCB boards: the control electronics board housing an FPGA which interfaces via $\mathrm{I} 2 \mathrm{C}$ to the onboard computer, with the data gathered from the plasma brake being small in comparison to the other payloads, less than $10 \mathrm{~KB}$ per $24 \mathrm{~h}$ of operation; a board for the tether and its reeling motor; and a third for housing the electron guns and necessary electronics, as well as some auxiliary electronics for the solar panels.

\section{Aalto-1 critical mission phases}

\subsection{Technology demonstration and science observations phase}

As seen in Fig. 5, before starting the science phase in which the payloads will perform their targeted science observations, the satellite will go through the commissioning phase. 


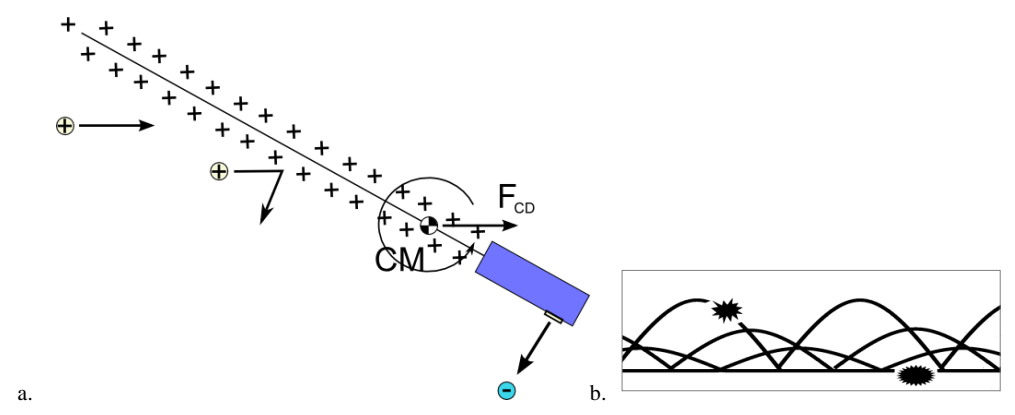

Fig. 4. (a) The situation with a positively charged tether; charged particles interacting with it, creating the Coulomb drag force $F_{\mathrm{CD}}$ parallel to the particle stream experienced by the whole spacecraft system (tether and spacecraft), while the tether itself is kept taut by the rotation of the spacecraft around its common centre of mass CM (not to scale). (b) Redundant strands ensure a level of tolerance to tearing (Seppänen et al., 2011).

\begin{tabular}{|l|l|}
\hline Boot - up and UHF/VHF Contact & S - band and Technology Demonstration \\
\hline
\end{tabular}

\begin{tabular}{|c|c|c|c|}
\hline Launch & Separation & Commissioning & Science Phase \\
\hline
\end{tabular}

Fig. 5. The commissioning phase will consist of booting up the satellite, making contact with ground and performing the required technical demonstration for the spectral imager and RADMON. The science phase is divided into two specific parts based on attitude and downlinking communication requirements.

During this phase, the satellite will be first booted up, contact made with the ground segment and pointed correctly towards nadir. After this has been achieved, spectral imager and RADMON technology operation will be demonstrated by taking 6 minimum quality spectral images and one VIScamera image, as well as operating the RADMON within the South Atlantic Anomaly (SAA) for altogether three days. This data will be sent down through the S-band downlink, and will serve at the same time as a way to test it as well.

The technology demonstration phase has no time limit. Once the technology demonstration goals have been met and in general the commissioning phase has gone according to plan, the science phase can begin. This phase is divided into two particular phases, as seen in Fig. 5, the first of which will consist of science observations performed with the spectral image and the RADMON, and the latter for operating the plasma brake. This division of the science phase is due to the drastically different attitude, data rate and power requirements of the different payloads. The science observations phase will last 6-12 months, depending on the satellite and payloads performance.
While performing the first part of the science phase, the satellite will assume either a nadir pointing or target tracking attitude with a velocity vector as seen in Fig. 1, and operate the spectral imager and the RADMON.

Both the spectral imager as well as the RADMON during this time will rely exclusively on the S-band downlink for transferring their collected data to the ground segment, and so are limited by how much it can possibly downlink during this time, especially the spectral imager. A preliminary estimate gives $29-49 \mathrm{MB}$ per $24 \mathrm{~h}$ for the S-band for an orbit altitude of $500-900 \mathrm{~km}$.

An average image size expected to be taken by the spectral imager is estimated to be around 7.8 MB $(512 \times 512$ pixels with 15 channels and 16 bits), which is for a minimum of 6 months science observations phase means being able to downlink around 623-1084 average images, depending again on the satellite orbit altitude. Thus, the spectral imager will take images several times per $24 \mathrm{~h}$, with a typical dynamical power usage profile as shown in Fig. 6 of a few minutes duration. 


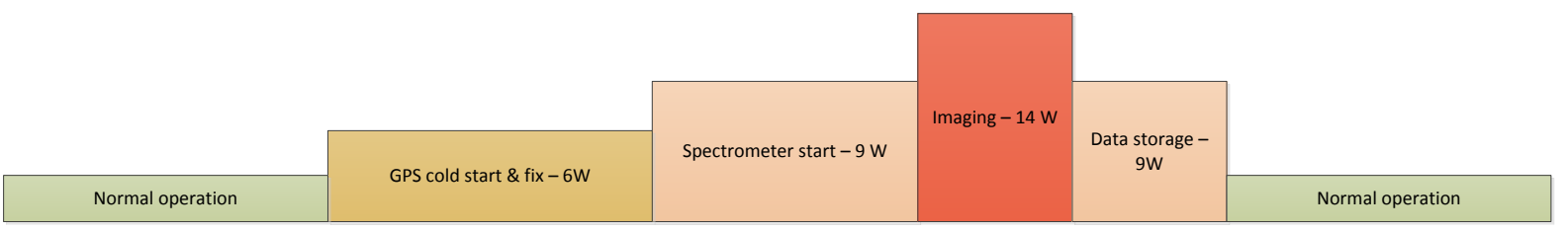

Fig. 6. The imaging sequence (not to timescale) with the spectral imager and associated total satellite power usage at an orbit height of $700 \mathrm{~km}$, the total time taking a few minutes for this whole sequence (GPS cold-start is by far the longest, the rest requiring around a second per operation block). As the spectral imager technology develops further, the power and time requirements presented here will most likely lessen. The power values here represent the total satellite power usage during a particular operation block within the imaging sequence.
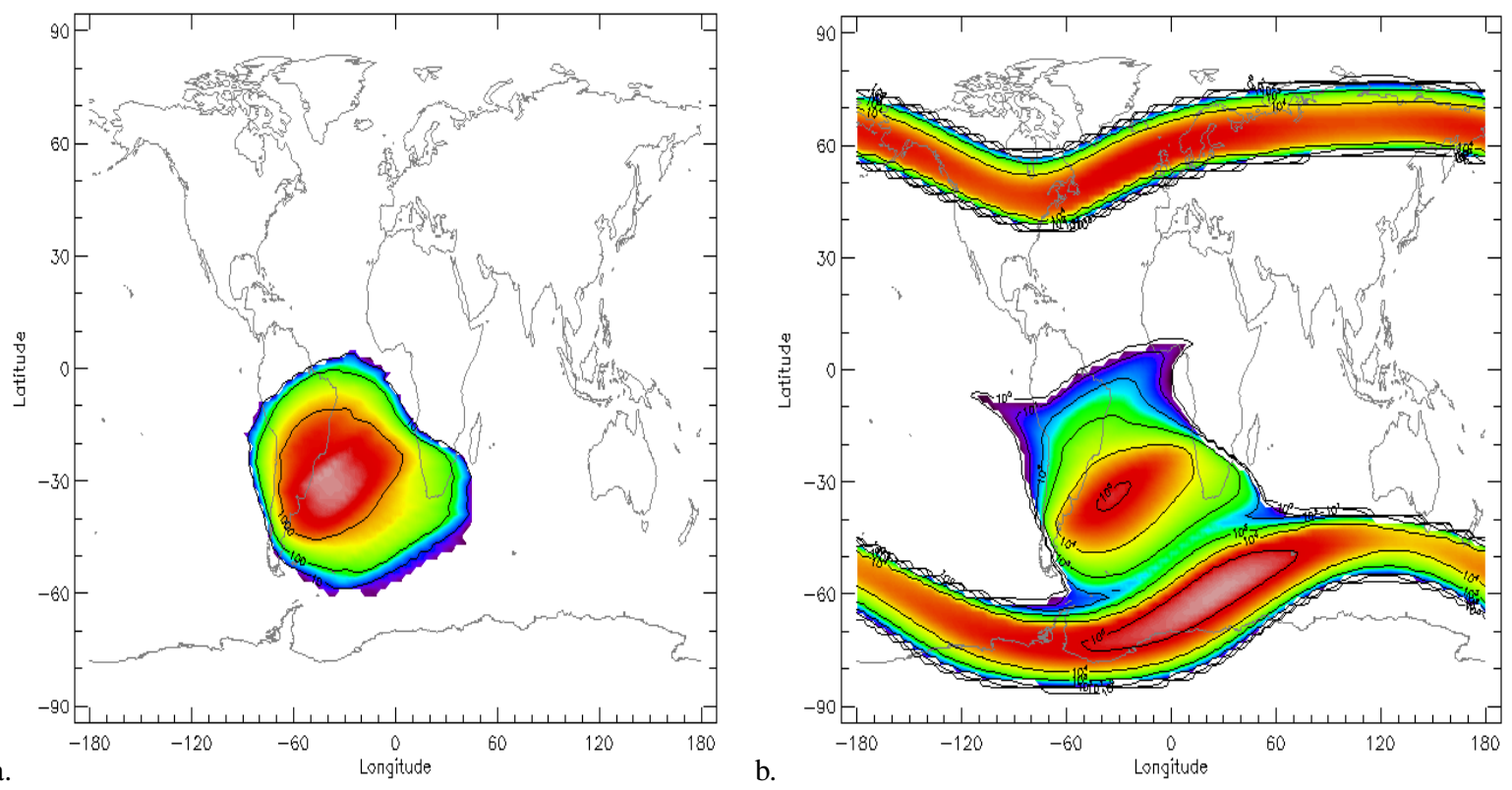

Fig. 7. Intended main targets for RADMON; (a) 10 or more $\mathrm{MeV}$ proton fluxes at a $700 \mathrm{~km}$ orbit height. (b) $700 \mathrm{keV}$ or more fluxes at the same height, produced by ESA Space Environment Information System (SPENVIS).

During this, the RADMON will be on $80 \%$ of the satellite's orbit period, switched off only during communication and spectral imager usage. It will have a constant perpendicular angle with respect to the nadir and the satellite's velocity vector, and will gather a steady average of around $2 \mathrm{MB}$ per $24 \mathrm{~h}$ when it is on, with main targets of interest for both proton and electrons being the planet's high latitudes and the SAA, as seen in Fig. 7.

The primary imaging targets are in Finland, both due to accessibility as well as good reference data availability. After the spectral imager technology has been demonstrated, VTT expects at least one datacube time-series to be imaged during the June-August growing season for the science observations phase. In addition, a "dark" image from the night side of the planet and another one from near the noon-equator will be taken to assess the minimum and maximum lighting performance of the imager.

Due to the uncertainty with the launch of the satellite and possible science observations phase duration of a whole year, separate targets have been chosen for the June-August growing season and for the winter season.

These targets leave plenty of data bandwidth unused; once both the technology demonstration targets and the abovementioned targets for the spectral imager have been met, a comprehensive campaign can be performed and is currently being planned.

Also a stereoscopic imaging campaign composed of several suitable targets around the planet is being planned.

\subsection{Electrostatic plasma brake demonstration}

The plasma brake will be operated separately in the second part of the science phase, the plasma brake demonstration phase. During this phase, the spectral imager will not be used due to drastically different satellite attitude parameters compared to the science observations phase, while the RADMON will be used sporadically and depending on the system power demands at that moment. 
Table 1. The electrostatic plasma brake demonstration phase steps.

\begin{tabular}{|c|c|c|}
\hline Event & $\begin{array}{r}\text { Average } \\
\text { power }\end{array}$ & $\begin{array}{l}\text { Time } \\
\text { taken }\end{array}$ \\
\hline 1. Satellite assumes a correct sun pointing attitude & $3.2 \mathrm{~W}$ & few minutes \\
\hline 2. Spin-up to $200^{\circ}$ per second & $2.6 \mathrm{~W}$ & $158 \mathrm{~h}$ \\
\hline 3. Tether reel-out to initial $10 \mathrm{~m}$ at $1 \mathrm{~mm} \mathrm{~s}^{-1}$ rate (EstCube-1 CubeSat length) & $5 \mathrm{~W}$ & $\sim 4 \mathrm{~h}$ \\
\hline 4. Initial experiment with $10 \mathrm{~m}$ of tether deployed in both positive and negative modes (with the radiation monitor on) & $5 \mathrm{~W}$ & 1 month \\
\hline 5. Reeling out the tether to $100 \mathrm{~m}$ full deployment length & $5 \mathrm{~W}$ & $\sim 34 \mathrm{~h}$ \\
\hline 6. Actual plasma brake experiment can begin, with the tether this time only in negative mode (with the radiation monitor on) & $5 \mathrm{~W}$ & $\sim 1 \mathrm{yr}$ \\
\hline 7. Continuing until the satellite has deorbited, once the primary experiment has been completed (optional) & $5 \mathrm{~W}$ & $\sim 1 \mathrm{yr}$ \\
\hline
\end{tabular}
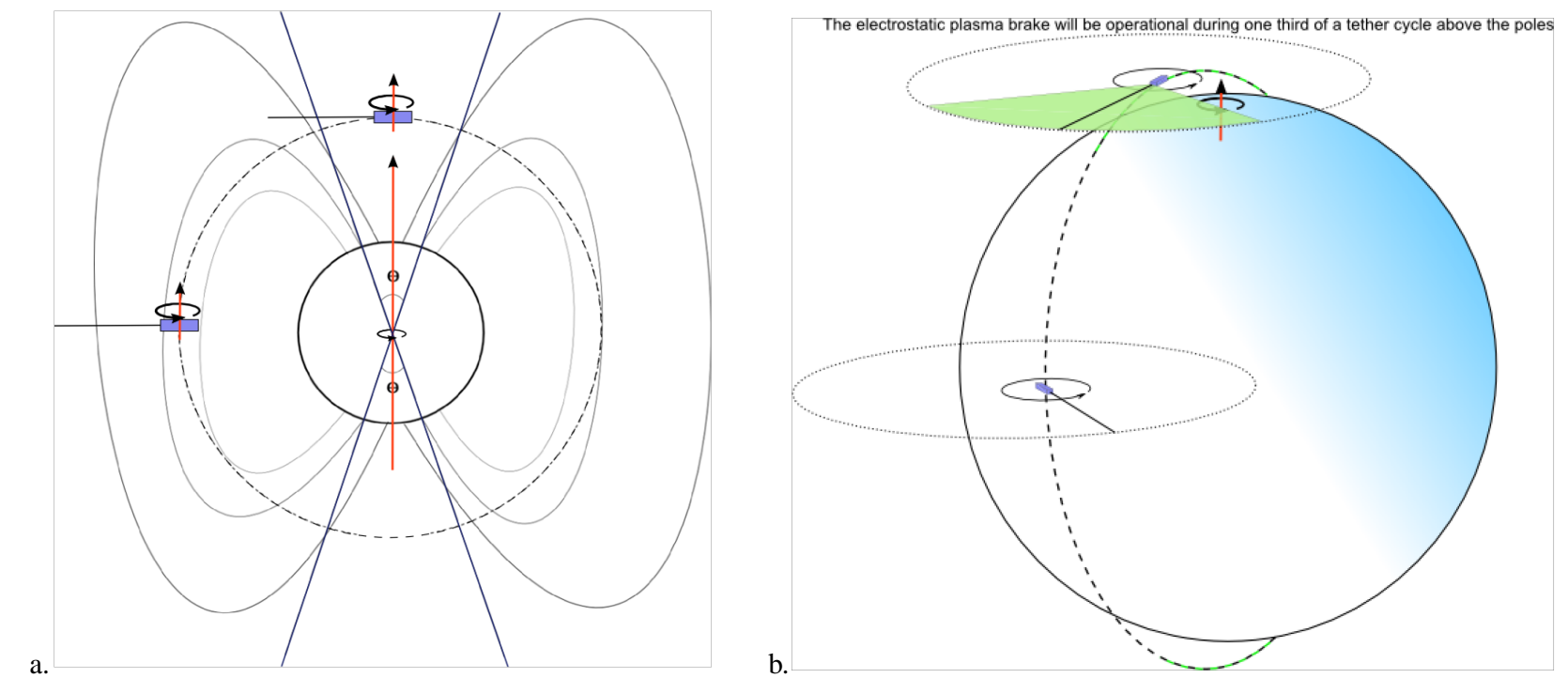

Fig. 8. (a) An example of the satellite moving in the magnetic field of the Earth. (b) Illustrating how the tether is active only in the vicinity of the poles (within an angle of $20^{\circ}$ ), marked as angle $\Phi$ in (a).

The experiment itself has the goal to demonstrate the technology behind the tether and how well it interacts with the LEO ionosphere around the planet. When moving with respect to the plasma of the ionosphere, it will create a Coulomb drag force due to the charged particles interacting with the equivalently charged tether, with the resulting force vector along the ionosphere velocity vector (see Fig. 4a). As the drag is exerted on the tether and so the rest of the satellite, it will discernibly change its orbit parameters by slowing it down. With regular position and velocity determination, such as GPS, the drag effect created by the ionosphere can, thus, be measured over the period of this last phase, and so the experiment performed.

Both positive and negative tether charging modes will be tested during this phase, as described in Table 1, and the negative mode requires only a voltage source and the tether itself, while the positive mode will need additionally $1-3$ electron guns to achieve the necessary charge levels in the tether; the tether in this mode gathers electrons from the plasma and shoots them out using the electron gun.
The major change of attitude dynamics in this part of the mission is due to the significantly different attitude requirements of the operational plasma brake tether. The satellite will be spun up to $200^{\circ}$ per second rotation around its own y-axis (see Fig. 1a) for keeping the tether taut during deployment, and the resulting rotation vector needs to be parallel to Earth's rotation vector, as shown in Fig. 8a. This is done to maximise the charged tether's interaction with the ionosphere, specifically in terms of relative velocity. For the same reason the tether will be charged and operational only during a $20^{\circ}$ total angle on top of the poles (see Fig. 8b). Similarly, for maximising the efficient use of Earth's magnetic during the satellite's spin up, as well as to conserve the power budget, the satellite will be spun up only during the same angle on top of the poles.

The initial tether deployment length is $10 \mathrm{~m}$, and the results of both positive and negative charged tether drag will be compared to the results of the cubesat EstCube-1, which has an almost identical mission and tether (but only $10 \mathrm{~m}$ long), and will be launched during 2013. The experiment will be performed with this initial length for roughly a month. After 
this, the satellite will be spun up again to $200^{\circ}$ per second and subsequently extended to its full length of $100 \mathrm{~m}$. This part of the plasma brake demonstration phase is scheduled to last up to a year in order to verify with certainty the effect, area and magnitude of the Coulomb drag force experienced by the charged tether. The tether will, however, be only negatively charged this time in order to not have to use the electron guns.

Once the phase and the mission has been completed, there is the option of continuing to slow down the satellite with the tether for enough time for it to drop altitude sufficiently and re-enter the atmosphere. This, however, depends on the initial orbit the launcher brings the satellite to, as well as how the mission itself works out and, therefore, is not a strictly mandatory goal. The radiation monitor will still be sporadically active during the demonstration, even though the satellite's rotation rate is very different in comparison to the science observations phase. It will see a $360^{\circ}$ view of the same orbit height as in the previous part of the science phase, with a comparatively sparser directional sampling rate. However, as the monitor's primary mission objective is by now complete, this will give additional, pseudo-3D data on the LEO radiation environment.

It is expected that the data rate per $24 \mathrm{~h}$ during this part of the science phase is no more than $10 \mathrm{~KB}$, with another 27.3 KB (with a sampling period of roughly $1 \mathrm{~min}$; this data amount includes the once per orbit GPS positioning for the same period) for housekeeping data via the UHF/VHF radios during the same period. As such, this means a drastically smaller required downlinking data rate, and as the new attitude rotation parameters effectively preclude the use of the S-band downlink, the UHF/VHF channels will be the main and only communication channels during the plasma brake demonstration.

\subsection{Expected positional and attitude errors influence on payload data}

The most sensitive payload to errors is the spectral imager. In turn, RADMON and the plasma brake are relatively insensitive, requiring attitude pointing of less than $10^{\circ}$, and attitude knowledge of one degree for RADMON and less than $10^{\circ}$ for the plasma brake. A positional accuracy of $10 \mathrm{~km}$ will be enough for both.

Table 2 summarises the largest error sources affecting the image itself (worst-case, without any post-processing assumed yet), with and without a GPS receiver. Due the varied nature of the reason for them, these errors will manifest as distortions and cut-offs of the image edges and so considering the image swath width is $120 \mathrm{~km}$ both along and crosstrack, will affect altogether $\sim 0.8 \mathrm{~km}(2 \times 0.4$ for opposite edges) the quality of the image in both track directions in the case GPS is used, while without GPS will be several kilometres possibly necessitating the auxiliary geometric correction using ground points. In both cases, however, attitude
Table 2. The estimates of various error sources on one image edge with and without inclusion of a GPS receiver. These are worst case values, without any post-processing assumed.

\begin{tabular}{lrl}
\hline Error sources & $\begin{array}{r}\text { With GPS } \\
\text { (worst case) }\end{array}$ & $\begin{array}{l}\text { Without GPS } \\
\text { (worst case) }\end{array}$ \\
\hline Attitude pointing & $0.290 \mathrm{~km}$ & $0.290 \mathrm{~km}$ \\
Tilt around line of sight & $0.02 \mathrm{~km}$ & $0.02 \mathrm{~km}$ \\
Satellite positioning & $0.1 \mathrm{~km}$ & $\begin{array}{l}\text { Varies depending on } \\
\text { backup method }\end{array}$ \\
\hline Total & $\sim 0.4 \mathrm{~km}$ & $\begin{array}{l}\text { Approximately the } \\
\text { positioning accuracy; } \\
\end{array}$ \\
& & up to several kilometres \\
\hline
\end{tabular}

and position subsystems are sufficient, and image degradation will be within payload requirements.

\subsection{Downlinking limitations influencing mission character}

The most dominating bottleneck on how the mission looks like is the downlinking bandwidth. The fact that UHF/VHF and S-band frequencies have been selected and only one ground station will be primarily available not only limits how much data can be sent down, but the opportunities for it as well. As the most interesting spectral imager mission targets will be in Finland (or close by), both communication with the ground station and imaging will have to be performed at the same time, causing relatively large peak power consumption. This will be demanding on the power budget of the satellite, and will so instead require prioritisation, either performing imaging on the spectral imager targets within the coverage area of the ground station or downlink communication. In practice, this means the required targets in the ground station coverage area should be imaged by imaging targets up to the maximum downlinking capability per $24 \mathrm{~h}$ and subsequently downlinking the image data, while for the rest of the mission priority is given to communication. TTC through UHF/VHF will be performed, however, during imaging as well. The RADMON will also be shut off during downlinking communication to conserve power.

When the plasma brake phase begins, this limitation will change form somewhat, as data downlinking and TTC will now be performed with UHF/VHF only, which requires less power. However, the spinning up of the satellite, as well as the operation of the tether, will occur within a total angle of $20^{\circ}$ over the poles, which will be partly in the coverage area of the ground station, necessitating to partially communication over plasma brake operation.

The RADMON will be shut off during communications and tether operation in order to make sure the gathered plasma brake and RADMON data as well as TTC within $24 \mathrm{~h}$ can be downlinked through the relatively narrow VHF. 


\section{Conclusions}

The Aalto-1 student nanosatellite project is rapidly developing forward, and the preliminary design phase has concretised into a clear system design, developing subsystem construction and testing, and most important, as a solid set of goals and a mission design. The skeleton of the Aalto1 science mission has been designed, with required targets for technical demonstration and operational procedures of the payloads developed. While every step of the actual science phase is not chosen yet, the framework with which the satellite will perform them has been designed and is being implemented.

The requirements of two drastically different parts of the science phase, the science observations phase and the plasma brake demonstration phase, have been taken into account and the operation designed according to the various bottlenecks that they cause, most important (and extensively interdependent) being attitude, power and downlinking communication bottlenecks. The downlinking communication and power budgets becomes especially constraining on the satellite when operating the spectral imager in the coverage area of the main, and only planned Aalto- 1 ground station.

The work presented here points to the direction that nanosatellites can not only perform technology demonstrations, but also a meaningful campaign of scientific observations with, compared to more traditional space missions, very limited resources.

The future effort in the satellite project will consist of the critical design phase; freezing the design, and actual building and testing of all satellite subsystems. The goal will be to have a flight-ready model, including the needed support equipment and the ground segment, ready by the end of 2013 and a targeted launch for the beginning of 2014.

Acknowledgements. Antti Kestilä acknowledges the financial support of the Helsinki University Graduate School of Atmospheric Composition and Climate Change.

Rami Vainio acknowledges the financial support of Magnus Ehrnrooth foundation and Vilho, Yrjö and Kalle Väisälä foundation.
Edited by: V. Korepanov

\section{References}

Antila, J., Miranto, A., Mäkynen, J., Laamanen, M., Rissanen, A., Blomberg, M., Saari, H., and Malinen, J.: MEMS and Piezo Actuator Based Fabry-Perot Interferometer Technologies and Applications at VTT, Proc. SPIE, 7680, 76800U, doi::978081948144-3, 2010.

CDS - Cubesat Design Specifications: CubeSat Design Specification Rev. 12, The Cubesat Program, Cal Poly SLO, http://cubesat.org/index.php/documents/developers, last access: 20 February 2013, California Polytechnic State University, California, 2009.

Janhunen, P., Toivanen, P. K., Polkko, J., Merikallio, S., Salminen, P., Haeggström, E., Seppänen, H., Kurppa, R., Ukkonen, J., Kiprich, S., Thornell, G., Kratz, H., Richter, L., Krömer, O., Rosta, R., Noorma, M., Envall, J., Lätt, S., Mengali, G., Quarta, A. A., Koivisto, H., Tarvainen, O., Kalvas, T., Kauppinen, J., Nuottajärvi, A., and Obraztsov, A.: Electric solar wind sail: Towards test missions, Rev. Sci. Instrum., 81, 111301-1-111301$11,2010$.

Mannila, R., Näsilä, A., Praks, J., Saari, H., and Antila, J.: Miniaturized spectral imager for Aalto-1 nanosatellite, Proc. SPIE, 8176, 817628-1-817628-8, doi:10.1117/12.897999, 2011.

Näsilä, A., Saari, H., Antila, J., Mannila, R., Kestilä, A., Praks, J., Salo, H., and Hallikainen, M.: Miniature Spectral Imager for the Aalto-1 Nanosatellite, Proccedings of the European CubeSat Symposium, Brussels, Belgium, 30 January 2012.

Saari, H., Aallos, V., Akujärvi, A., Antila, T., Holmlund, C., Kantojärvi, U., Mäkynen, J., and Ollila, J.: Novel Miniaturized Hyperspectral Sensor for UAV and Space Applications, Proc. SPIE, 7474, 74741M-1-74741M-12, doi:10.1117/12.830284, 2009.

Seppänen, H., Kiprich, S., Kurppa, R., Janhunen, P., and Haeggström, E.: Wire-to-wire bonding of micrometer-diameter aluminium wires for the Electric Solar Wind Sail, Microelect. Eng., 88, 3267-3269, 2011.

US Patent US 8, 130, 380 B2: Spectrometer and Interferometric Method, Heikki Saari, VTT, http://www.uspto.gov/, last access: February 2013, United States Patent and Trademark Office, Alexandria, USA, 6 March 2012. 